\title{
Synthesis, Structural and Mechanical Characterization of Amorphous and Crystalline Boron Nanobelts
}

\author{
Hai Ni and Xiaodong $\mathrm{Li}^{*}$ \\ Department of Mechanical Engineering, University of South Carolina \\ 300 Main Street, Columbia, South Carolina 29208, USA \\ ”lixiao@engr.sc.edu (corresponding author)
}

Received February 22, 2007; accepted May 252007

\begin{abstract}
Keywords: Boron; Nanobelts; Transmission electron microscopy; Nanoindentation; Atomic force microscopy
\end{abstract}

\begin{abstract}
Amorphous and crystalline (rhombohedral structure with [111] growth direction) boron nanobelts were synthesized by the vapor-liquid-solid technique. Their structure and chemical compositions were studied by various electron and atomic force microscopy techniques. Most amorphous and crystalline belts have a width to thickness ratio of 2 and are covered with a layer of amorphous silicon oxide. The crystalline belt cores are defect-free single crystals. Gold catalyst thickness and synthesis temperature are the two prominent parameters determining structure of the synthesized nanobelts. The elastic modulus and hardness were measured using nanoindentation and atomic force microscopy three-point bending techniques. The indentation elastic modulus and hardness were measured to be $92.8 \pm 4.5 \mathrm{GPa}$ and $8.4 \pm 0.6$ GPa for amorphous belts, and 72.7 $\pm 3.9 \mathrm{GPa}$ and $6.8 \pm 0.6 \mathrm{GPa}$ for crystalline ones, respectively. The three-point bending elastic moduli were found to be $87.8 \pm 3.5 \mathrm{GPa}$ and $72.2 \pm 2.4 \mathrm{GPa}$ for amorphous and crystalline, respectively. The measured mechanical properties are 4-5 times lower than those of the counterpart bulk materials.
\end{abstract}

\section{Introduction}

Boron, one spot left to carbon in the Periodic Table, is a unique element with three-electrondeficient in nature [1-3]. It tends to form unique three-centered covalent bonds with a shorter radius that induce several icosahedral cluster-based crystalline structures, such as $\alpha$ - and $\beta$ rhombohedral boron, and $\alpha$-tetragonal boron. The crystal structure diversity of boron renders it to be one of the most fascinating materials with a variety of superior properties. As is well known, bulk form boron has a high melting temperature near $2300^{\circ} \mathrm{C}$, high elastic modulus (380-400 GPa) [4,5] and high hardness (Knoop: $2160-2900 \mathrm{~kg} / \mathrm{mm}^{2}$ or 21.2-28.4 GPa) close to that of diamond. It also has low moderate oxidation resistance, interesting optical and electrical properties, and low thermal conductivity [2].

Stimulated by these intriguing properties of bulk boron, researchers have made great effort in synthesizing one dimensional (1D) boron nanomaterials utilizing a variety of techniques, such as radio-frequency magnetron sputtering [6], chemical vapor deposition (CVD) [7,8], laser ablation technique [9-11], and carbothermal reactions [12,13]. Such 1D boron nanomaterials are expected to possess various bulk properties, and they may serve as nanobuilding blocks for the next generation sensors, electric devices, vacuum microelectronics, nano/microelectromechanical systems (NEMS/MEMS) and other high-temperature electronics. They also have the promise to be used in applications such as nanocomposites where they may work as excellent strengtheners, imparting stiffness, toughness, and strength.

Another impetus in synthesizing 1D boron nanomaterials is to manufacture superconducting 
1D $\mathrm{MgB}_{2}$ nanostructures. Since the discovery of superconducting $\mathrm{MgB}_{2}$, there has been great interest in direct synthesis of $1 \mathrm{D} \mathrm{MgB}_{2}$ nanomaterials. However, direct synthesis still faces great challenges and the only available route so far to achieve $\mathrm{MgB}_{2}$ nanowires is to diffuse $\mathrm{Mg}$ into pure boron nanowires $[14,15]$. During such an ex situ process, boron nanowires serve as the precursor for the $\mathrm{MgB}_{2}$ nanowires to be formed via $\mathrm{Mg}$ diffusion. Therefore, to grow boron nanowires is a prerequisite in order to obtain superconducting $\mathrm{MgB}_{2}$ nanowires [7].

As compared with other 1D nanomaterial systems, such as carbon [16,17], $\mathrm{ZnO}$ [18-21] and $\mathrm{GaN}$ [22-24], physical properties of 1D boron nanomaterials and especially their mechanical properties have rarely been studied. In order to integrate $1 \mathrm{D}$ boron nanomaterials into functional nanodevices, their mechanical properties and structural integrity are of paramount importance. Mechanical failures that occur at the nanobuilding block interfaces may cause malfunction or even break down of the entire nanodevices [25-30]. Therefore, a precise measurement of the mechanical properties of nanostructures is greatly needed.

In this study, amorphous and crystalline boron nanobelts were first synthesized by CVD via a vapor-liquid-solid mechanism. Their structure and compositions were then characterized by electron microscopy and atomic force microscopy techniques. Nanoindentation experiments were performed directly on individual belts to measure their mechanical properties, such as the elastic modulus and hardness, and to study indentation deformation behavior. In addition, three-point bending tests were also performed on individual nanobelts to study their elastic moduli using atomic force microscopy techniques.

\section{Experimental Details}

Nanobelt Synthesis and Characterization. The synthesis of both amorphous and crystalline boron nanobelts was carried out in a torch-sealed quartz tube heated in a three-zone horizontal tube furnace [31]. Boron, silicon and iodine powders (weight ratio 40:1:1) were mixed, compacted, and loaded into a smaller quartz boat, and then placed to one end of a quartz tube (3/4 inch in diameter) as source materials. Two long stripes of single crystal Si (100) substrate, sputter-coated with 20 and $3 \mathrm{~nm}$ thick gold (for amorphous and crystalline nanobelts, respectively), were positioned at the other end of the quartz tube. The quartz tube was torchsealed with a base pressure of $5 \mathrm{mTorr}$, and then was loaded into the tube furnace with the source materials position at the center of the furnace. The average heating rate was $45^{\circ} \mathrm{C} / \mathrm{min}$. Once the temperature was raised to $1150^{\circ} \mathrm{C}$, the furnace was held for $20 \mathrm{~min}$ for substrate with $20 \mathrm{~nm}$ gold catalyst for amorphous nanobelts and $115 \mathrm{~min}$ for substrate with $3 \mathrm{~nm}$ gold catalyst for crystalline nanobelts. After holding at $1150^{\circ} \mathrm{C}$ for the desired growth time, the torch sealed quartz tube was pulled out from the furnace and cooled in air to room temperature. The morphology and chemical composition analyses of the as-synthesized boron nanomaterials were performed by scanning electron microscopy (SEM, FEI Quanta 200) and energy dispersive X-ray (EDX) spectroscopy, transmission electron microscopy (TEM, Hitachi H-8000), high resolution (HR) TEM (Titan 80-300 TEM), EDX and parallel electron energy loss spectroscopy (TEMEDX/PEELS), and atomic force microscopy (AFM, DI 3100, Veeco Metrology Group). The grown boron nanomaterials were detached from the substrate into high-purity methanol solvent to form nanomaterial suspension by sonicating for 15-30 seconds. TEM and HRTEM samples were prepared by dropping $\sim 10 \mu 1$ suspension onto lacy $\mathrm{Cu}$ TEM grids.

Nanoindentation. A Hysitron Triboscope nanoindenter (Hysitron, Inc.) with in situ scanning capability was used to perform nanoindentation tests directly on individual nanobelts. The nanoindenter monitored and recorded the load and displacement of the three-sided pyramidal diamond (Berkovich) indenter during indentation with a force resolution of about $1 \mathrm{nN}$ and a displacement resolution of about $0.2 \mathrm{~nm}[29,30]$. The indenter tip was used to locate and image individual boron nanobelts. By adjusting the trace and retrace profiles, the scanned area can be gradually decreased to final $1-3 \mathrm{~nm}^{2}$, with the center of the nanobelt high profile located in the center. After the image stabilized, an indentation was made on the nanobelt in situ using the same 
diamond indenter and the impression was then imaged with the same tip as well. Post-test imaging provides the ability to verify if the test was performed in the anticipated location, which maximizes the reliability of data and aids in explanation of unexpected test results. Hardness and elastic modulus were calculated from the load displacement data obtained by nanoindentation using the well-accepted Oliver-Pharr method [32]. In order to compensate the indenter geometry effect due to wear in service on the measurement accuracy, tip geometry calibration or area function calibration was carefully carried out, on a regular basis, on a standard quartz plate with known elastic modulus and hardness of 69.8 and 9.09 GPa. Furthermore, before each nanoindentation test, the thermal drift was automatically tracked and recorded by means of introducing the nanoindenter in touch with the top surface of the sample with minimum contact load. All the nanoindentation tests were performed when the thermal drift or vibration induced mechanical drift dropped to $0.01 \mathrm{~nm} / \mathrm{s}$. The load displacement curves were obtained by subtracting the drift effect for hardness and elastic modulus analyses. The details of the nanoindentation theories can be found elsewhere [29,30,32].

AFM Three-point Bending. A Veeco Dimension 3100 AFM was used to perform three-point bending tests on both amorphous and crystalline nanobelts by directly indenting the center of a suspended single nanobelt with a diamond tip having a known spring constant of $195 \mathrm{~N} / \mathrm{m}$. Both types of nanobelts were bridged over the $1 \mu \mathrm{m}$ wide trenches via dropping the above solution onto a standard AFM 3D reference sample (3D reference, Veeco Metrology Group). The detailed procedures can be found in other publications $[18,22,33]$.

\section{Results and Discussion}

Morphology, Chemical and Structural Characterization. The structure and morphology of both amorphous and crystalline boron nanobelts are illustrated in Fig. 1. Fig. 1a presents typical low and high (insert) magnification SEM images, showing a dense and thick film of boron nanobelts on a silicon (100) substrate sputter coated with $20 \mathrm{~nm}$ gold catalyst. These nanobelts were collected at a temperature zone of $1050-1075^{\circ} \mathrm{C}$, which is around $75^{\circ} \mathrm{C}$ lower than the maximum holding temperature of $1150^{\circ} \mathrm{C}$. Although the synthesizing temperature is higher than $1100^{\circ} \mathrm{C}$ and the sample was quenched in air, crystalline boron nanobelts were not achieved on a large potion of the substrate, except at the higher temperature zone of around $1100^{\circ} \mathrm{C}$. This experiment proved that in addition to the high cooling rate, a temperature gradient is another important factor to achieve crystalline structure in boron nanobelts. Figs. $1 \mathrm{~b}$ and $1 \mathrm{c}$ are low and high magnification TEM bright-field images of nanobelts collected at $1050-1075^{\circ} \mathrm{C}$ of the intermediate temperature zone. The width of the nanobelts revealed by TEM is in the range from 100 to $700 \mathrm{~nm}$ and a couple of hundreds of micrometers long. The insert in Figure $1 \mathrm{~b}$ is a selected area electron diffraction pattern (SAD) from the boron nanobelts. The diffusive ring pattern clearly shows that the as-synthesized boron nanobelts are of amorphous characteristic.

Crystalline boron nanobelts were also obtained on a silicon (100) substrate with thinner (3 nm) gold catalyst, at a temperature zone of $1100^{\circ} \mathrm{C}$, which is only $50^{\circ} \mathrm{C}$ lower than the maximum holding temperature of $1150^{\circ} \mathrm{C}$. As compared to the thicker catalyst, shown in Fig. 1a, the thinner catalyst results in less denser nanobelts as shown in Fig. 1d. Figs. 1e and 1f are low and high magnification TEM bright-field images of the crystalline nanobelts. SAD pattern (insert in Fig. 1e) indicates that the as-synthesized boron nanobelts are single crystal with the rhombohedral structure (space group $R \overline{3} m$ ). 
The morphological characteristics of both amorphous and crystalline nanobelts were also confirmed by AFM, as shown in Figs. $2 a$ and $2 b$, respectively. The AFM section analysis shows that most studied amorphous nanobelts are in the range of 100-400 nm high and $200-800 \mathrm{~nm}$ wide with a width to height ratio close to 2 . For crystalline boron nanobelts, the size shrinks to 50-200 $\mathrm{nm}$ high and 100-400 $\mathrm{nm}$ wide with almost the same width to height ratio as the amorphous nanobelts. For both amorphous and crystalline cases, in addition to belt-like morphology, small numbers of wires were occasionally observed as well.
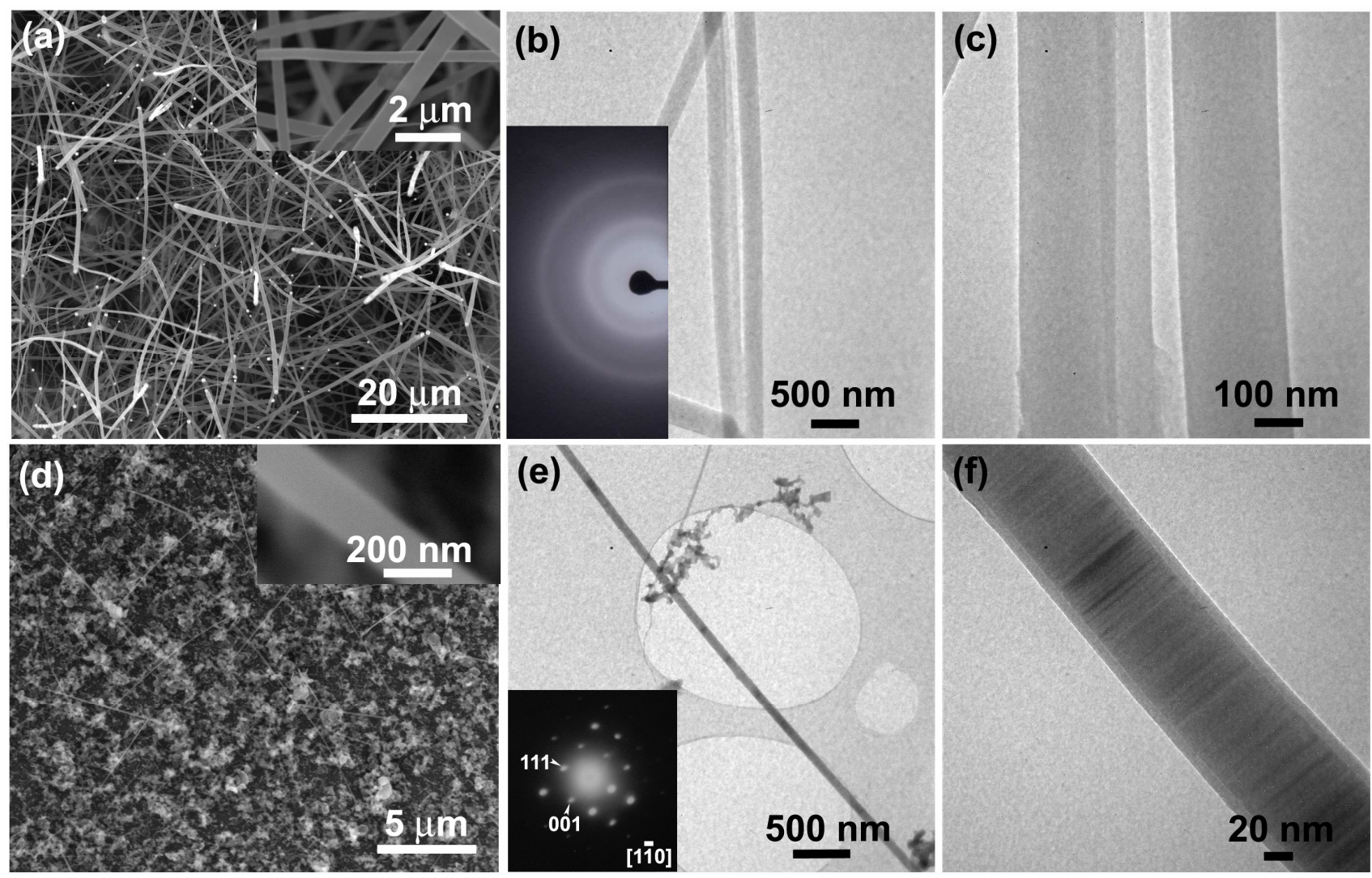

Fig. 1. (a) SEM image showing the as-synthesized, dense amorphous boron nanobelts on Si (100) substrate coated with $20 \mathrm{~nm}$ thick $\mathrm{Au}$. Insert is a high magnification SEM image showing the nanobelt morphology. (b) and (c) Low and high magnification TEM bright-field image showing the morphology of amorphous boron nanobelts. Insert in (b) is the SAD pattern showing a diffusive ring pattern of amorphous structure. (d) SEM image showing the as-synthesized, sparse boron nanobelts on silicon (100) substrate coated with $3 \mathrm{~nm}$ thick Au. (e) Low magnification TEM brightfield image showing the morphology of a crystalline boron nanobelt. The SAD pattern (insert) was

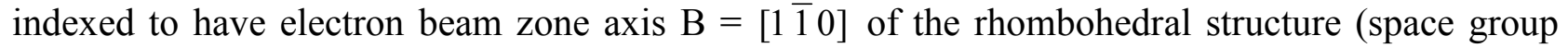
$R \overline{3} \mathrm{~m}$ ). (f) High magnification TEM bright-field image showing the nanobelt has a defect free crystalline core covered with an amorphous surface layer of a thickness of $5 \sim 7 \mathrm{~nm}$.

In order to qualitatively characterize their chemical compositions, HRTEM-EDX and PEELS studies were also performed on both amorphous and crystalline nanobelts and the results are presented in Figs. 3 and 4, respectively. For amorphous nanobelts, the EDX result in Fig. 3a clearly shows that there is a strong boron peak, together with a strong silicon peak and a weak oxygen peak. The inserts in Fig. 3a are a TEM image, which shows the area where the EDX was collected from, and the corresponding SAD pattern, which confirms the amorphous nature. To eliminate the side effect from the TEM copper 
grid on the chemical composition analysis, EDX analysis was also carried out on the adjacent carbon film and the result is shown in Fig. 3b. As compared with Fig. 3a, one can firmly conclude that the as-synthesized amorphous nanobelts consist of boron, silicon and oxygen, in which oxygen and silicon exist as a thin layer oxide covering the boron core. The PEELS spectra shown in Fig. 3c also confirm the presence of boron. Similar EDX and PEELS results were also obtained from crystalline boron nanobelts, as shown in Figs. 4c, 4d and 4e. The atomic lattice of a crystalline nanobelt is revealed in the HRTEM image shown in Fig. 4a. The insert in Fig. 4a is the fast Fourier transform (FFT) image. The crystalline boron core is covered by a layer of amorphous silicon oxide with a thickness variation up to a few nanometers. The SAD pattern shown in Fig. 4b agrees well with the above conclusion about the rhombohedral lattice structure. These crystalline boron nanobelts have a growth direction along [111], and the measured distance between (111) lattice planes is $8.2 \AA$. The strips around the diffraction spots
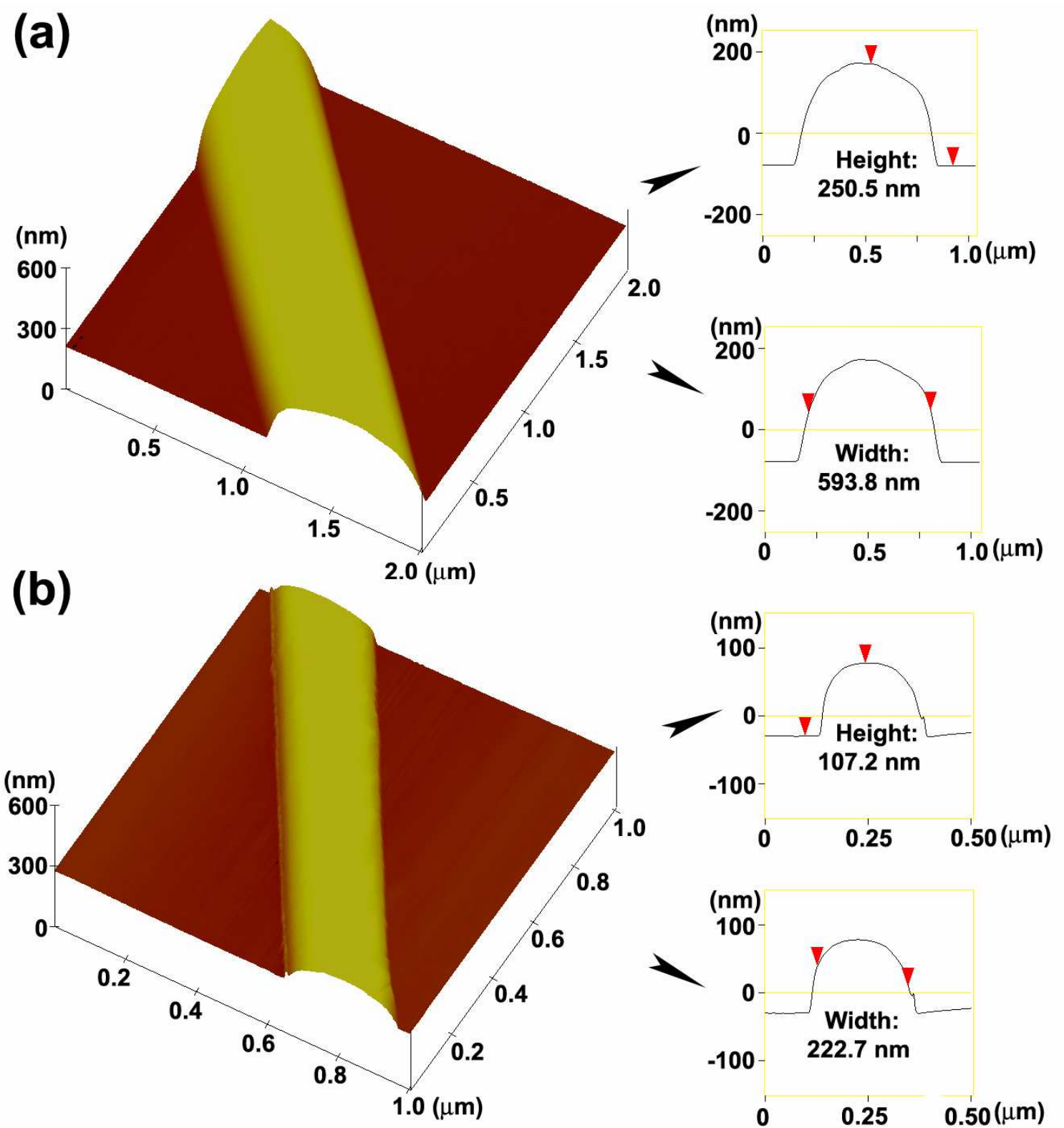

Fig. 2. 3D AFM images and section analysis showing the morphological characteristics and width and height profiles of both (a) amorphous and (b) crystalline boron nanobelts. 
are believed to be due to the fine lattice structure, and this further resulted in the extended boron peak as one can see on both PEELS spectra.

The formation of the silicon oxide layer, which covers the boron nanobelts, can be understood on the basis of $\mathrm{Si}$ and $\mathrm{O}$ vapor pressure levels [31]. At the early stage of synthesis, the gold
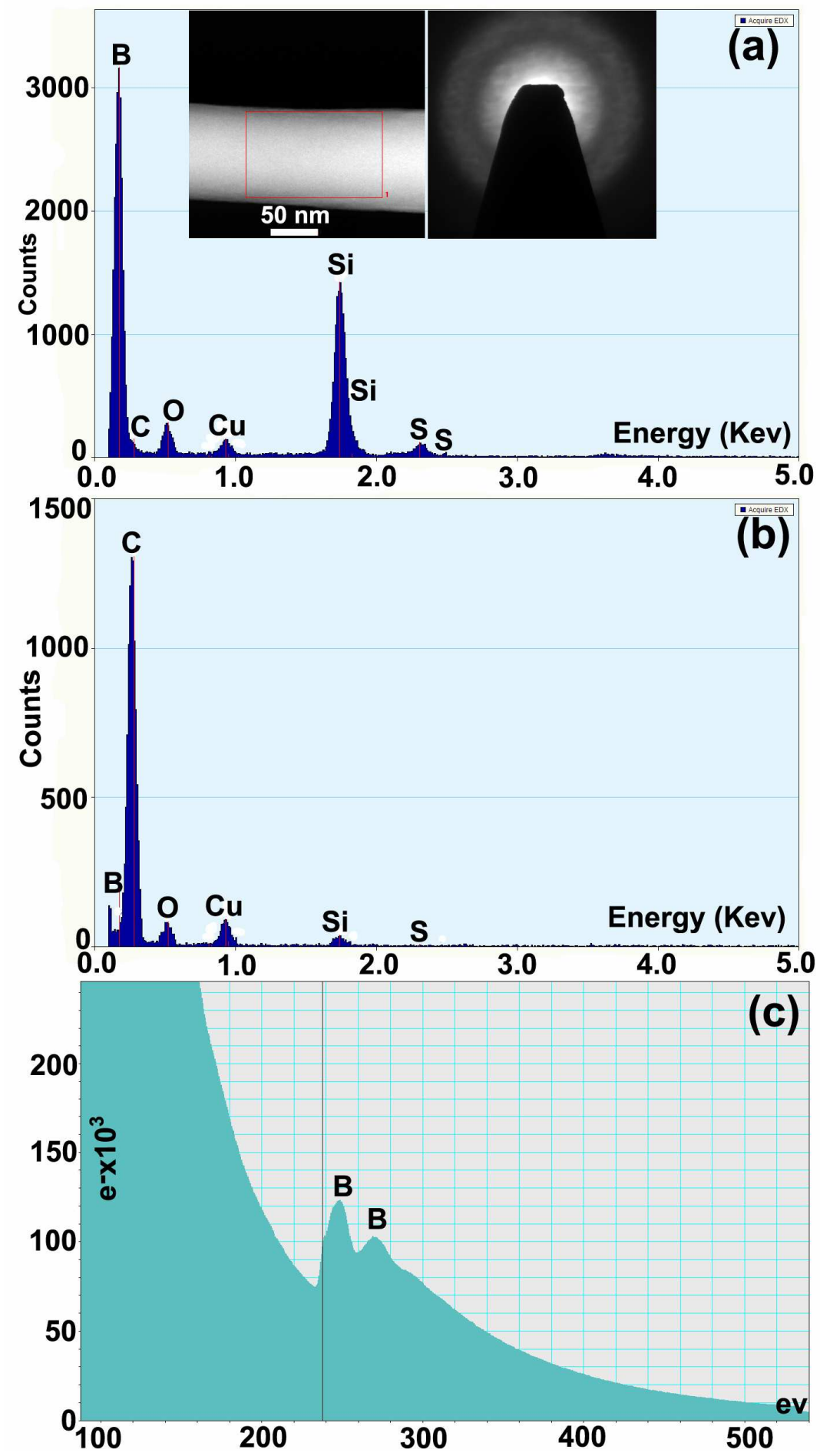

Fig. 3. TEM/EDX spectra from (a) amorphous boron nanobelts (inserts are a TEM image and the SAD), and (b) adjacent carbon film on $\mathrm{Cu}$ grid. (c) PEELS spectrum. 
form small islands and absorbs mainly boron atoms to initiate the growth of boron nanobelts. As the growth proceeds, the vapor pressures of both $\mathrm{Si}$ and $\mathrm{O}$ continue to increase reaching a threshold value, and thus simultaneously trigger the formation of a thin layer of silicon oxide on the boron belt surface.
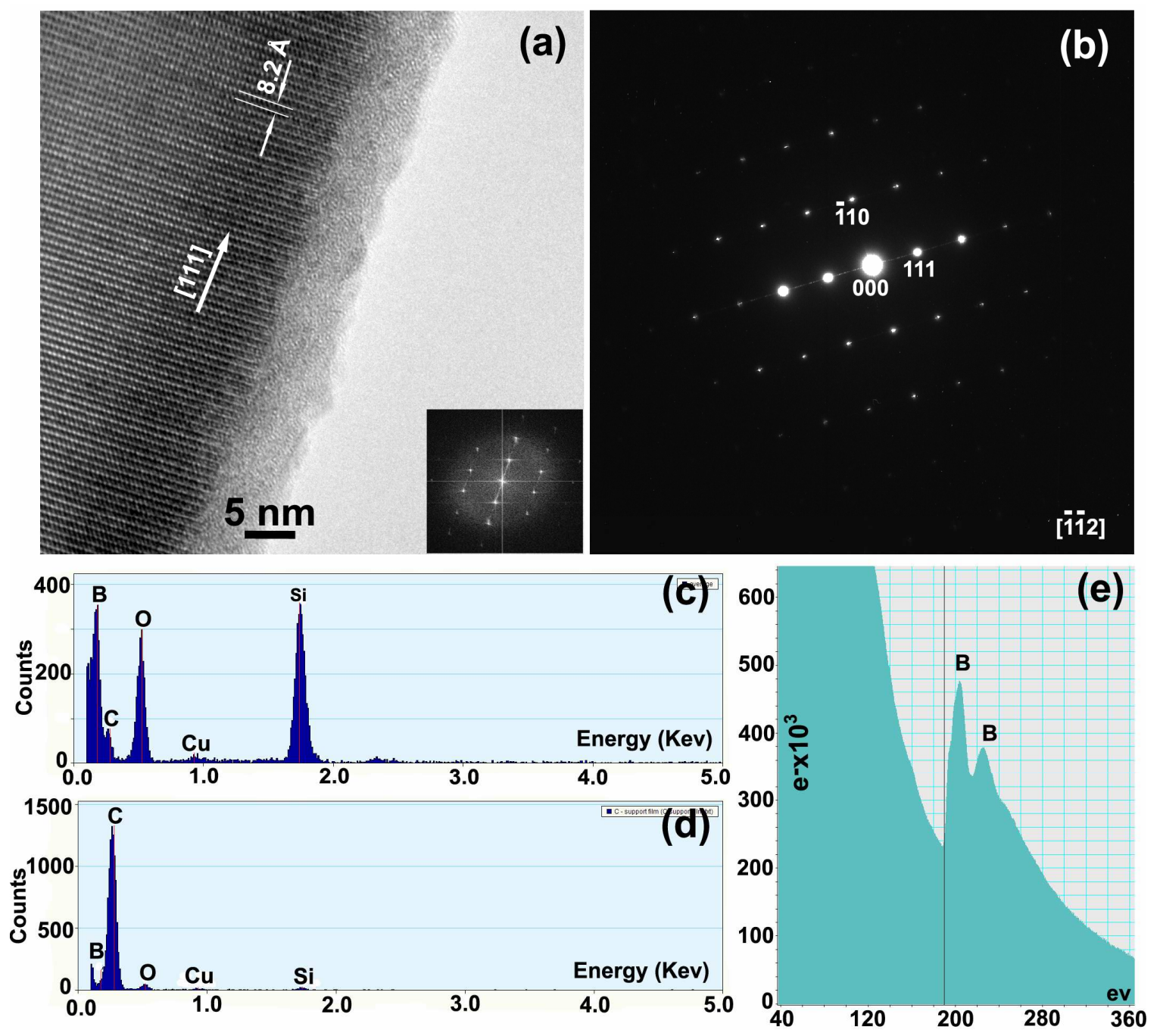

Fig. 4. (a) HRTEM image showing the atomic structure of the crystalline boron core covered with the amorphous silicon oxide surface layer. The growth direction is along [111]. Insert is the corresponding fast fourier transform image. (b) The SAD pattern with electron beam zone axis $\mathrm{B}=\left[\begin{array}{ll}-1-1 & 2\end{array}\right]$ of the rhombohedral structure (space group $R \overline{3} \mathrm{~m}$ ). TEM/EDX spectra from (c) crystalline boron nanobelts, and (d) adjacent carbon film on $\mathrm{Cu}$ grid. (e) PEELS spectrum.

Elastic Modulus and Hardness Measured by Nanoindentation. Representative loaddisplacement curves and indentation impressions on both amorphous and crystalline nanobelts are illustrated in Fig. 5. For indentation studies of both amorphous and crystalline boron nanobelts, two different loading schemes, shown as inserts in Figures $5 \mathrm{a}$ and 5b, were employed. A peak indentation load of $150 \mu \mathrm{N}$ was applied to the indenter with and without a peak load-holding segment of 4 seconds. Both loading and unloading rates were kept the same at $25 \mu \mathrm{N} / \mathrm{s}$. As shown in Figures 5a, for amorphous boron nanobelts, a certain amount of permanent deformation $(\sim 8 \mathrm{~nm})$ was generated at room temperature after 

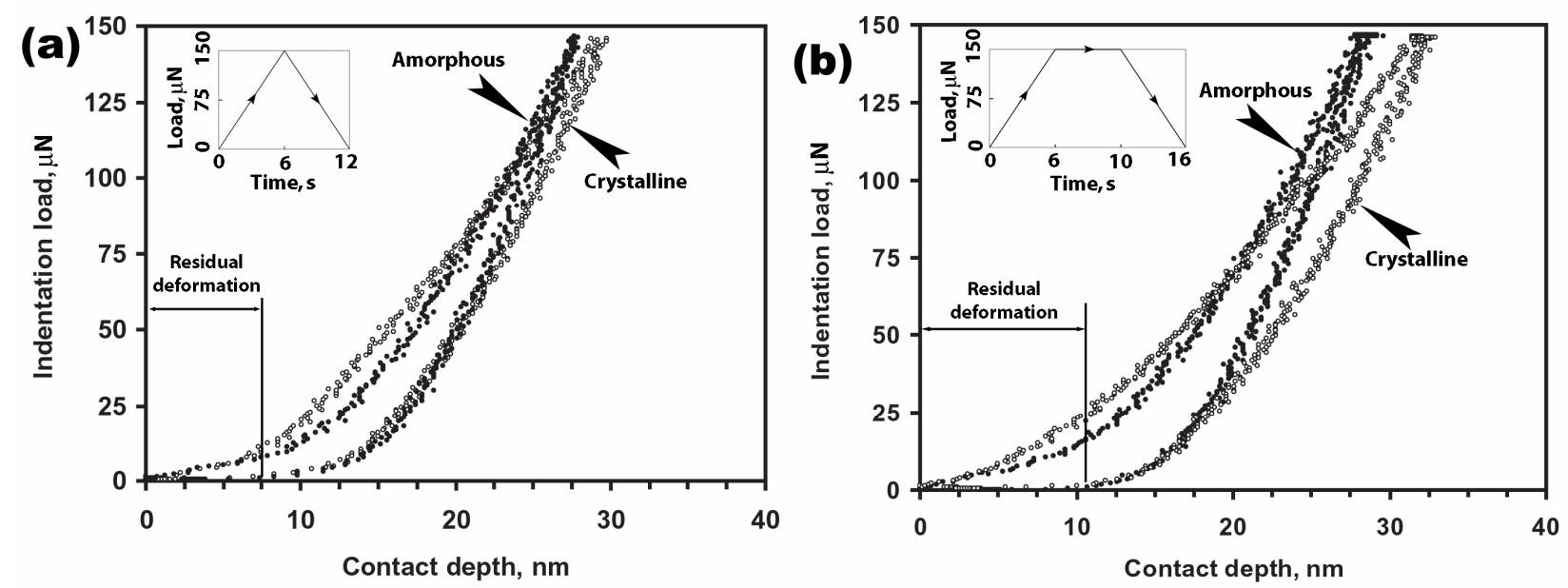

(c)

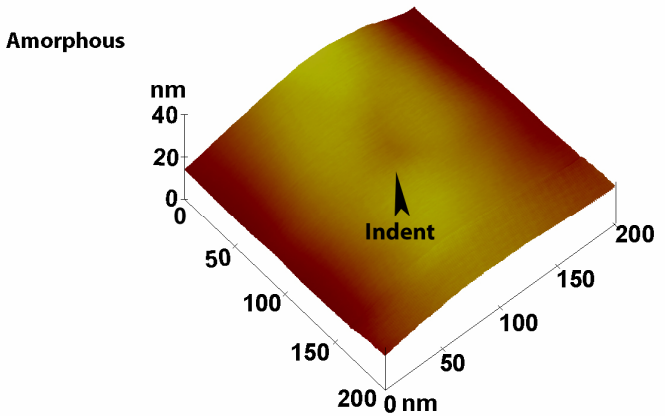

(d)

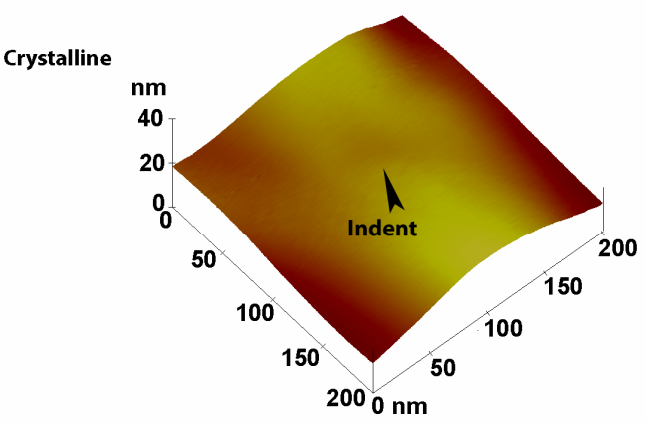

Fig. 5. Nanoindentation load-displacement curves without (a) and with (b) peak indentation load holding segment for both amorphous and crystalline boron nanobelts showing a significant amount of residual deformation of crystalline boron after the peak indentation load was removed. (c) and (d) 3D AFM images showing the nanoindentation impressions made on the amorphous and crystalline nanobelts, respectively, at a peak indentation load of $400 \mu \mathrm{N}$.

the peak indentation load was removed. This fact indicates that both types of boron nanobelts show ductile characteristic at room temperature. Figs. $5 \mathrm{c}$ and $5 \mathrm{~d}$ show the indent marks made on the amorphous and crystalline boron nanobelts, respectively, at a higher indentation load of $400 \mu \mathrm{N}$. Moreover, when the peak indentation load was removed after holding for 4 seconds, a larger amount of permanent deformation (around $11 \mathrm{~nm}$ ) was observed for both amorphous and crystalline belts as shown in Fig. 5b. Furthermore, Fig. 5b also shows that for both types of belts there is almost negligible amount of creep (less than $2 \mathrm{~nm}$ ) generated during the holding segment of 4 seconds at the peak indentation. This phenomenon indicates that both amorphous and crystalline boron nanobelts have better room temperature creep resistant than $\mathrm{ZnS}$ and $\mathrm{ZnO}$ nanomaterial systems [33,18] and hence may work as excellent nanobuilding blocks and interconnects, where room temperature creep is critical. For all nanoindentation tests, the maximum indentation depth was strictly controlled to about $30 \%$ of the thickness of each studied nanobelt. By doing this, both the substrate effect and the surface oxide layer effect on elastic modulus and hardness were kept to a minimum.

The measured elastic modulus and hardness of both amorphous and crystalline boron nanobelts as a function of contact depth are shown in Fig. 6a. The elastic modulus of amorphous boron nanobelts is $92.8 \pm 4.5 \mathrm{GPa}$, whereas that of crystalline ones is $72.7 \pm 3.9 \mathrm{GPa}$. It is very clear that the elastic modulus for both amorphous and crystalline boron nanobelts are much lower (4 times) than that of counterpart bulk materials, which is $380 \sim 400 \mathrm{GPa}[4,5]$. The low elastic modulus, as compared to the counterpart bulk materials, was also observed in other 1D nanomaterials, such as 
$\mathrm{ZnO}[18,20], \mathrm{ZnS}$ [34] and $\mathrm{GaN}$ [22]. As shown in Fig. 6b, the average hardness values for amorphous and crystalline boron nanobelts are $8.4 \pm 0.6 \mathrm{GPa}$ and $6.8 \pm 0.6 \mathrm{GPa}$, which are 2.5 and 3.1 times lower than that of bulk boron, respectively.
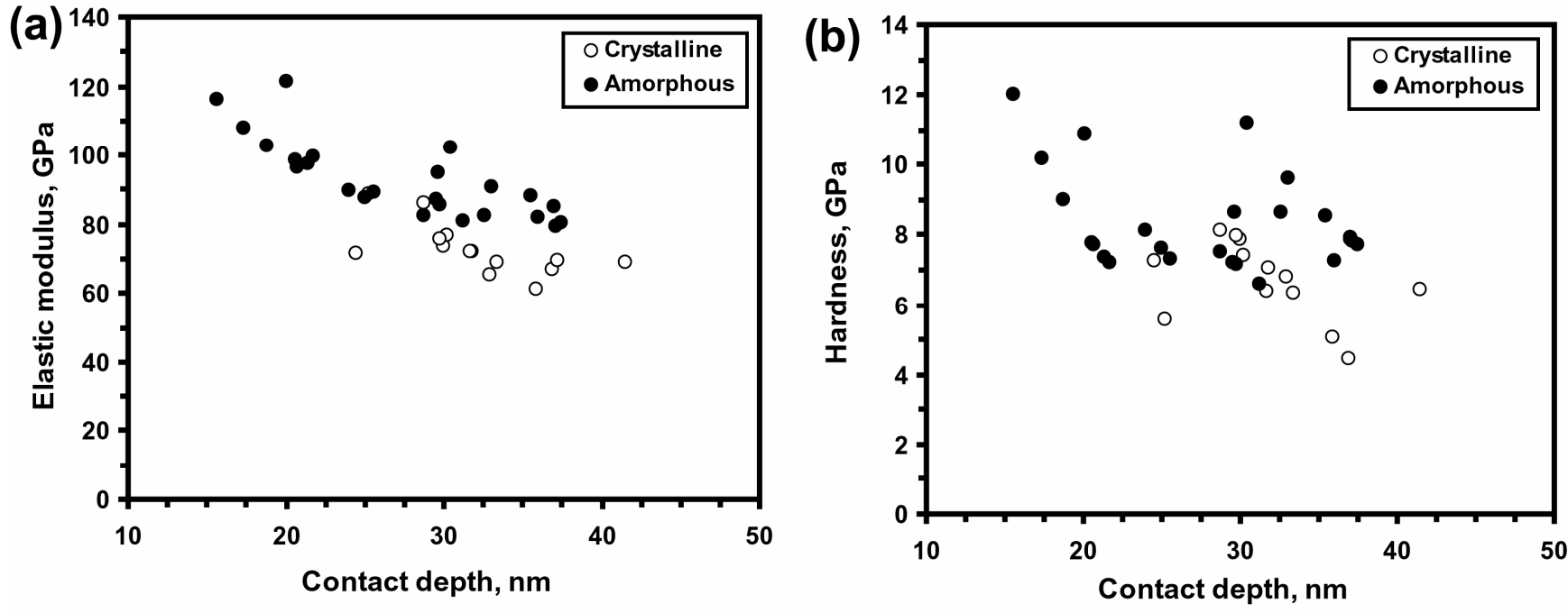

Fig. 6. (a) Elastic modulus and (b) hardness of both amorphous and crystalline boron nanobelts as a function of indentation contact depth, respectively.

Elastic Modulus Measured by AFM Three-point Bending. In order to make comparable studies between the mechanical properties obtained through different testing methods, AFM three-point bending tests were also carried out on both types of boron nanobelts, and the results are depicted in Fig. 7. Fig. 7a shows a typical loading-unloading curve in terms of AFM applied force (nN) and piezo position along $\mathrm{z}$ direction, obtained from a typical suspended crystalline nanobelt under a nominal applied force of 2 Volts. It should be noted that the measured total deflection $\left(\mathrm{d}_{\mathrm{t}}\right)$ consists of the deflection from the cantilever $\left(\mathrm{d}_{\mathrm{c}}\right)$ and the deflection from the nanobelt $\left(\mathrm{d}_{\mathrm{n}}\right)$. The real applied load $(\mu \mathrm{N})$ and nanobelt deflection $(\mathrm{nm})$ curve can be further obtained by subtracting the deflection of the cantilever $\left(\mathrm{d}_{\mathrm{c}}\right)$ from the total measured deflection $\left(\mathrm{d}_{\mathrm{t}}\right)$, and by multiplying the cantilever spring constant $(195 \mathrm{~N} / \mathrm{m})$ with the recorded applied force in $\mathrm{nN}$. Fig. 7b shows such curves between AFM applied load and net deflection of a single nanobelt of both amorphous and crystalline. It is clear that the AFM applied load and nanobelt deflection keeps very good linear relationship within the applied load of $60 \mu \mathrm{N}$.

In order to extract the bending elastic modulus of both amorphous and crystalline nanobelts, the spring constant of each nanobelt has to be known. The spring constant of a nanobelt $\left(k_{n}\right)$ is defined as the slope of the initial linear potion of the AFM applied load and nanobelt deflection curve. According to the linear elastic theory of an isotropic material, the elastic modulus of a nanobelt, $E_{n}$, can be calculated from Eq. 1 [25]

$$
E_{n}=F L^{3} / 192 d_{n} I=k_{n} L^{3} / 192 I \text {. }
$$

where $I$ is the moment of inertia. For a rectangle shape nanobelt, $I=b h^{3} / 12$, where $b$ and $h$ are the width and height of the nanobelt, respectively. $L$ is the suspended length of the nanobelt and $F$ is the applied load at its mid-point position. $k_{n}\left(=F / d_{n}\right)$ is the spring constant of the nanobelt. The bending elastic modulus of both amorphous and crystalline boron belts are shown in Fig. $7 \mathrm{c}$ and are measured to be $87.8 \pm 3.5 \mathrm{GPa}$ and $72.2 \pm 2.4 \mathrm{GPa}$, respectively, which are slightly lower than the corresponding nanoindentation ones as illustrated in Fig. $7 \mathrm{~d}$ and Table 1. These measured mechanical properties, much lower than that of counterpart bulk material, will be very critical to the 
manufacturing of various nanodevices when boron nanobelts are used as building blocks or interconnects, since mechanical properties of bulk boron cannot be directly transferred to the 1D boron nanomaterials.

Table 1. Summary of elastic modulus and hardness values of amorphous and crystalline boron nanobelts measured by nanoindentation and AFM three-point bending techniques.

\begin{tabular}{|c|c|c|c|}
\hline & $\begin{array}{c}\text { Indentation } \\
\mathrm{E}[\mathrm{GPa}]\end{array}$ & $\begin{array}{c}\text { Indentation } \\
\mathrm{H}[\mathrm{GPa}]\end{array}$ & $\begin{array}{c}\text { AFM Bending } \\
\mathrm{E}[\mathrm{GPa}]\end{array}$ \\
\hline Amorphous & $92.8 \pm 4.5$ & $8.4 \pm 0.6$ & $87.8 \pm 3.5$ \\
\hline Crystalline & $72.7 \pm 3.9$ & $6.8 \pm 0.6$ & $72.2 \pm 2.4$ \\
\hline
\end{tabular}
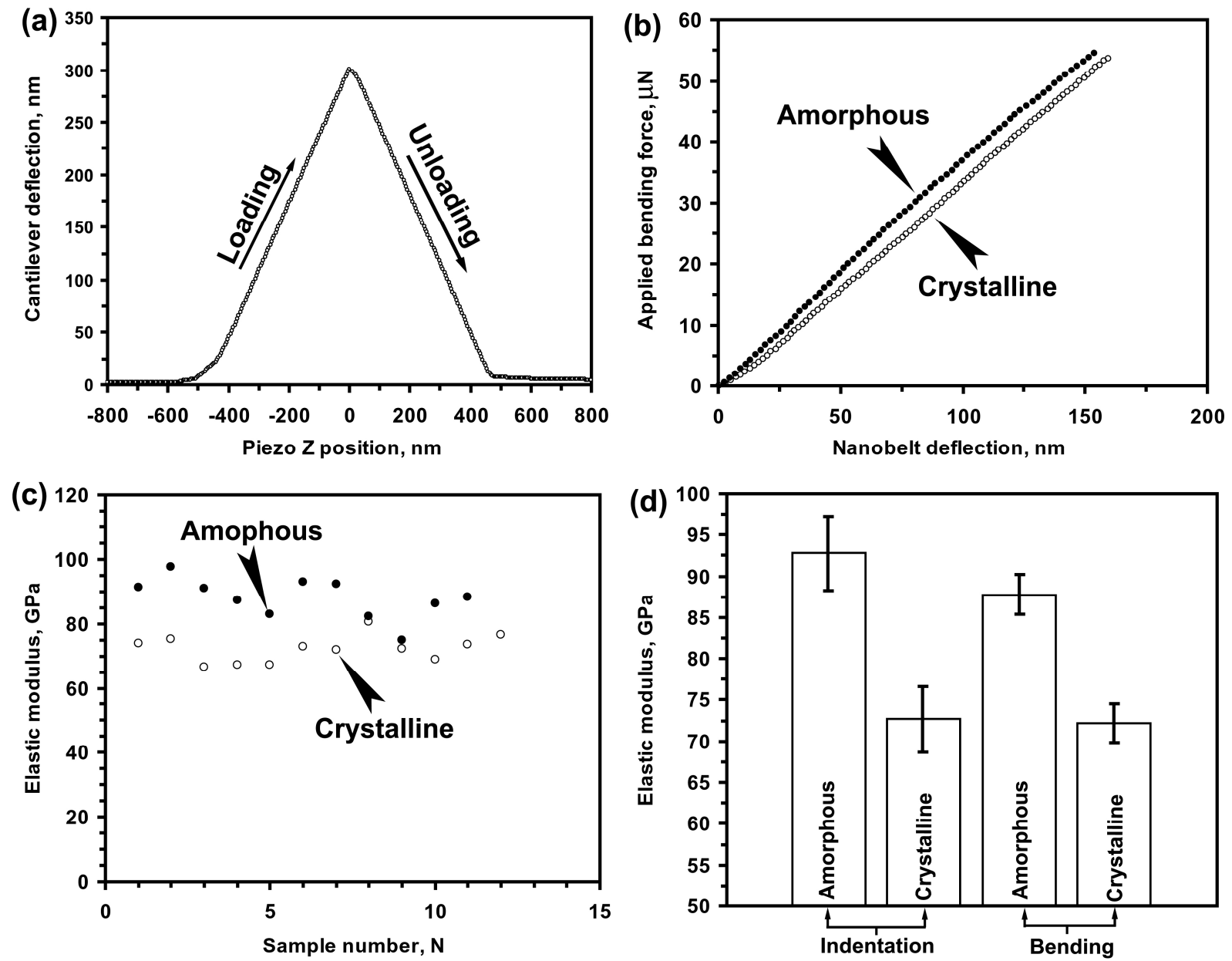

Fig. 7. (a) AFM recorded applied force (unfolded) in unit of $\mathrm{nm}$ as a function of piezo $\mathrm{z}$ traveling position. (b) AFM applied bending force $(\mu \mathrm{N})$ as a function of net nanobelt deflection $(\mathrm{nm})$ of both amorphous and crystalline belts under a nominal load of $60 \mu \mathrm{N}$ (only loading portion is shown). (c) Bending elastic moduli of both amorphous and crystalline nanobelts. (d) Elastic modulus and hardness comparison between amorphous and crystalline nanobelts obtained by nanoindentation and AFM three-point tests. 


\section{Conclusions}

In summary, we have synthesized amorphous and crystalline boron nanobelts using a vapor-liquidsolid technique. Both amorphous and crystalline belts have a width to thickness ratio of 2 and are covered with a layer of amorphous silicon oxide. The crystalline belt cores are defect-free single crystals of the rhombohedral structure with the belt growth direction in the [111] orientation. Gold catalyst thickness and synthesis temperature are the two prominent parameters determining the structure of the synthesized nanobelts (amorphous or crystalline). The elastic modulus and hardness for amorphous and crystalline boron nanobelts obtained from both nanoindentation and AFM three point bending agree well with each other, but are found to be much lower than those of bulk boron.

\section{Acknowledgments}

The authors would like to thank all staff members at the University of South Carolina Electron Microscopy Center, Henk Colijn at the Ohio State University Campus Electron Optics Facility and members in Nanostructures and Reliability Laboratory at the University of South Carolina for SEM, TEM and nanoindentation technical support. The authors would also like to acknowledge the financial supports from the National Science Foundation (CMMI-0653651 and EPS-0296165), the ACS Petroleum Research Fund (ACS PRF\# 40450-AC10), and the University of South Carolina NanoCenter.

\section{References}

[1] V. I. Matkovich (Eds), Boron and Refractory Boride, Springer-Verlag, Berlin, 1977.

[2] R.M. Adams, Ed., Boron, Metallo-Boron, Compounds and Boranes, Interscience Publishers, New York, 1964.

[3] N.N. Greenwood, A. Earnshaw, Chemistry of the Elements, Reed Educational and Professional Publishing Ltd, UK, 1997.

[4] J.E. Bailey, in Handbook of Polymer-Fibre Composites, F.R. Jones (Eds.), Longman Scientific \& Technical, Harlow, UK, 1994.

[5] F.N. Tavadze, J.V. Lominadze, A.F. Khvedelidze, G.V. Tsagareishvili, M.K. Shorshorov, S.I. Bulichev, The effect of impurities on the mechanical properties of zone melted boron, J. LessCommon Met. 82 (1981) 95-97.

[6] L. Cao, Z. Zhang, G. Li, J. Zhang, W. Wang, Well-aligned boron nanowire arrays, Adv. Mater. 13 (2001) 1701-1704.

[7] Y. Wu, B. Messer, P. Yang, Superconducting $\mathrm{MgB}_{2}$ nanowires, Adv. Mater. 13 (2001) 14871489.

[8] J.Z. Wu, S.H. Yun, A. Dibos, D.K. Kim, M. Tidrow, Fabrication and characterization of boron-related nanowires, Microelectr. J. 34 (2003) 463-470.

[9] Y.J. Zhang, H. Ago, M. Yumura, T. Komatsu, S. Ohshima, K. Uchida, S. Iijima, Synthesis of crystalline boron nanowires by laser ablation, Chem. Commun. 23 (2002) 2806-2807.

[10] Y.J. Zhang, H. Ago, M. Yumura, S. Ohshima, K. Uchida, T. Komatsu, S. Iijima, Study of the growth of boron nanowires synthesized by laser ablation, Chem. Phys. Lett. 385 (2004) 177183.

[11] X.M. Meng, J.Q. Hu, Y. Jiang, C.S. Lee, S. T. Lee, Boron nanowires synthesized by laser ablation at high temperature, Chem. Phys. Lett. 370 (2003) 825-828.

[12] Q. Yang, J. Sha, J. Xu, Y.J. Ji, X.Y. Ma, J.J. Niu, H.Q. Hua, D. R. Yang, Aligned single crystal boron nanowires, Chem. Phys. Lett. 379 (2003) 87-90. 
[13] T.T. Xu, J.G. Zheng, N.Q. Wu, A.W. Nicholls, J.R. Roth, D.A. Dikin, R.S. Ruoff, Crystalline boron nanoribbons: synthesis and characterization, Nano Lett. 4 (2004) 963-968.

[14] S. Jin, H. Mavoori, C. Bower, R. B. van Dover, High critical currents in iron-clad superconducting $\mathrm{MgB}_{2}$ wires, Nature 411 (2001) 563-566.

[15] P.C. Canfield, D.F. Finnermore, S.L. Bud'ko, J.E. Ostenson, G. Lapertot, C.E. Cunningham, C. Petrovic, Superconductivity in dense MgB2 wires, Phys. Rev. Lett. 86 (2001) 2423-2426.

[16] R.S. Ruoff, D. Qian, W.K. Liu, Mechanical properties of carbon nanotubes: theoretical predictions and experimental measurements Comptes rendus Physique 4 (2003) 993-1008.

[17] E.W. Wong, P.E. Sheehan, C.M. Lieber, Nanobeam mechanics: Elasticity, strength, and toughness of nanorods and nanotubes, Science 277 (1997) 1971-1975.

[18] H. Ni, X. D. Li, Young's modulus of $\mathrm{ZnO}$ nanobelts measured using atomic force microscopy and nanoindentation techniques, Nanotechnology 17 (2006) 3591-3597.

[19] G. Feng, W.D. Nix, Y. Yoon, C. J. Lee, A study of the mechanical properties of nanowires using nanoindentation, J. Appl. Phys. (2006) 074304.

[20] S. Mao, M. Zhao, Z.L. Wang, Nanoscale mechanical behavior of individual semiconducting nanobelts, Appl. Phys. Lett. 83 (2003) 993-995.

[21] X.D. Bai, P.X. Gao, Z.L. Wang, E. D. Wang, Dual-mode mechanical resonance of individual ZnO nanobelts, Appl. Phys. Lett. 82 (2003) 4806-4808.

[22] H. Ni, X.D. Li, G. S. Cheng, R. Klie, Mechanical properties of single-crystal GaN nanowires, J. Mate. Res. 21 (2006) 2882-2887.

[23] E. Stern, G. Cheng, E. Cimpoiasu, R. Klie, S. Guthrie, J. Klemic, I. Kretzschmar, E. Steinlauf, D. Turner-Evans, E. Broomfield, J. Hyland, R. Koudelka, T. Boone, M. Young, A. Sanders, R. Munden, T. Lee, D. Routenberg, M. A. Reed, Electrical characterization of single GaN nanowires, Nanotechnology 16 (2005) 2941-2944.

[24] G. Fasol, Room-temperature blue gallium nitride laser diode, Science, 272 (1996) 1751-1752.

[25] X.D. Li, B. Bhushan, Fatigue studies of nanoscale structures for MEMS/NEMS applications using nanoindentation techniques, Surf. Coat. Technol. 163-164 (2003) 521-526.

[26] X.D. Li, B. Bhushan, K. Takashima, C.W. Baek, Y.K. Kim, Mechanical characterization of micro/nanoscale structures for MEMS/NEMS applications using nanoindentation techniques, Ultramicroscopy 97 (2003) 481-494.

[27] X.D. Li, B. Bhushan, Nanofatigue studies of ultrathin hard carbon overcoats used in magnetic storage devices, J. Appl. Phys. 91 (2002) 8334-8336.

[28] X.D. Li, B. Bhushan, Development of a nanoscale fatigue measurement technique and its application to ultrathin amorphous carbon coatings, Scripta Mater. 47 (2002) 473-479.

[29] X.D. Li, B. Bhushan, A review of nanoindentation continuous stiffness measurement technique and its applications, Mater. Charact. 48 (2002) 11-36.

[30] X.D. Li, B. Bhushan, Nanomechanical characterisation of solid surfaces and thin films, Int. Mater. Rev. 48 (2003)125-164. ,

[31] H. Ni, X.D. Li, Self-assembled composite nano-/micronecklaces with $\mathrm{SiO}_{2}$ beads in boron strings, Appl. Phys. Lett. 89 (2006) 053108. 
[32] W.C. Oliver, G.M. Pharr, An improved technique for determining hardness and elasticmodulus using load and displacement sensing indentation experiments, J. Mater. Res. 7 (1992) 1564-1583.

[33] H. Ni, X.D. Li, H.S. Gao, Elastic modulus of amorphous $\mathrm{SiO}_{2}$ nanowires, Appl. Phys. Lett. 88 (2006) 043108.

[34] X.D. Li, X.N. Wang, Q.H. Xiong, P.C. Eklund, Mechanical properties of ZnS nanobelts, Nano Lett. 5 (2005)1982-1986. 